\title{
Evidence of Ionization Blue Shift Seeding of Forward Raman Scattering
}

\author{
Shouyuan Chen*, Ping Zhang*, Wolfgang Theobald*, Ned Saleh*, Matthew \\ Rever*, Anatoly Maksimchuk* and Donald Umstadter*
}

${ }^{*}$ FOCUS Center, University of Michigan, Ann Arbor, MI, 48109.

\begin{abstract}
.
We report on the results of spectroscopic experiments that were conducted by focusing an intense ultra-short laser pulse onto a helium gas target. The scattered light from the interaction region was measured spectrally and spatially from various directions as a function of laser intensity and plasma density. The experimental data showed that forward Stimulated Raman Scattering (SRS) was sensitive to the focus position of laser relative to the nozzle. Together with the plasma channel that was imaged by a CCD camera, the measurements indicate that SRS is seeded by the ionization blue-shifted light. The cross-phase modulation between the SRS and laser beam was also observed in the experiment.
\end{abstract}

\section{INTRODUCTION}

With recent development of ultrashort, high power laser, the laser intensities can easily exceed $10^{17} \mathrm{Wcm}^{-2}$. Such high laser intensities can suppress the Coulomb barrier of electrons in atoms and cause rapid tunnelling ionization. This leads to many interesting nonlinear phenomena in the laser gas interaction, such as laser frequency blue-shift by the copropagating ionization front[1], ionization defocusing[2] and harmonic generation[3]. Recently, experimental results and simulation from virous research groups indicate that ionization process also plays an important role in the stimulated Raman scattering (SRS) in laser-plasma interaction as a seeding mechanism [4][5][6].

The SRS in the plasma is a parametric processes in which a photon $(\omega, \vec{k})$ from an incident laser beam interact with electron perturbation in a plasma wave and decays to a lower frequency photon $\left(\omega_{p}, \vec{k}-\overrightarrow{k_{p}}\right)$ and a plasmon $\left(\omega_{p}, \overrightarrow{k_{p}}\right)$ or absorbers a plasmon and is upshifted to higher frequency photon $\left(\omega+\omega_{p}, \vec{k}+\vec{k}_{p}\right)$, which are called Stokes and anti-Stokes frequencies, respectively. To trigger the SRS, an optical signal at Raman wavelength should present at the beginning of the laser-plasma interaction. Generation of this signal is called the seeding mechanism. There are several proposed seeding mechanisms. One is the Raman backscattering seeding mechanism[7], in which the Raman signal grows from the thermal noise generated by the ponderomotive force of the laser pulse in the plasma. Another proposed seeding mechanism is the blue shift of the laser frequency from ionization front[4], which is the ionization self-steepening in time domain. In this mechanism, the Raman signal is generated from the blue-shift of the laser frequency by the ionization front. We report on experimental evidence for the latter mechanism in this paper. 


\section{Experimental Setup}

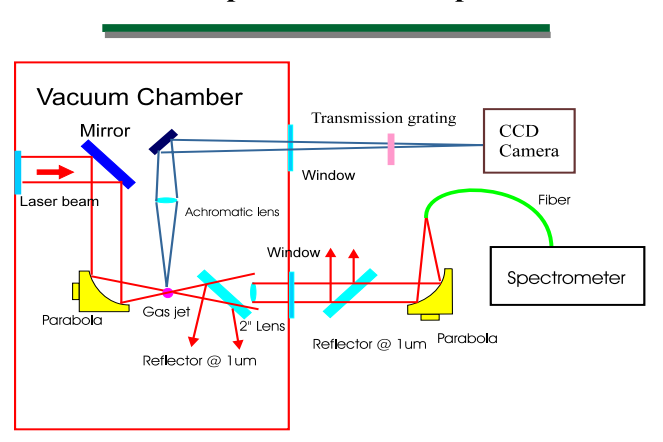

FIGURE 1. The experimental setup.

\section{EXPERIMENT}

The experiment was conducted with a $400 \mathrm{fs,} \mathrm{P}$ polarized, Ti-sapphire/Nd-glass hybrid laser system with a $1.053 \mu \mathrm{m}$ laser wavelength at the University of Michigan FOCUS center. The 2-inch laser beam was focused by an f/3 parabola onto the edge of a helium gas target generated by a $1.2 \mathrm{~mm}$ diameter supersonic nozzle. The backing pressure of the solenoid valve was 800 PSI. The focused laser spot diameter was 12 $\mu \mathrm{m}$ FWHM, which contains $60 \%$ of the laser energy. In the experiment, the laser power was varied from $0.45 \mathrm{TW}$ to $2.4 \mathrm{TW}$. Accordingly, the peak intensity was varied from $2.4 \times 10^{17} \mathrm{~W} / \mathrm{cm}^{2}$ to $1.3 \times 10^{18} \mathrm{~W} / \mathrm{cm}^{2}$. The experimental setup is illustrated in Figure 1. A 2-inch diameter lens with a $30 \mathrm{~cm}$ focal length was used to collimate the light propagating in the forward direction (collection solid angel $\Omega=1.8 \mathrm{msr}$ ) and then a parabolic mirror focused the collimated light to the Ocean Optics Spectrometer, which has a detection range from $650 \mathrm{~nm}$ to $1100 \mathrm{~nm}$ with a resolution of $1.3 \mathrm{~nm}$. In order to improve the contrast of the forward Raman satellite with the fundamental laser light, two high reflectors at laser wavelength are used to reflect the laser beam, and various combinations of neutral filters are put in front of the spectrometer to further reduce the light intensity. The laser plasma interaction was monitored from the top of the gas jet. The spectral resolved topview image was obtained by placing a transmission grating in front of the topview CCD camera.

Figure 2 shows the spectra of the laser pulse and forward SRS measured at a laser power of $0.45 \mathrm{TW}$. The plasma frequency measured from the frequency shift was $0.28 \times 10^{15} \mathrm{~s}^{-1}$, and the corresponding plasma density was $2.45 \times 10^{19} \mathrm{~cm}^{-3}$. The measured Raman spectrum bandwidth was $3.8 \mathrm{~nm}$, which was slightly larger than the laser bandwidth of $3.2 \mathrm{~nm}$. Figure 3 shows the forward Raman spectra when the gas target moved relative to the focus position. The Raman signal was strongest when the laser was focused at the edge of the gas jet and disappear when the focus position moved $100 \mu \mathrm{m}$ inside. With increased laser intensity, the intensity of the Raman signal was 


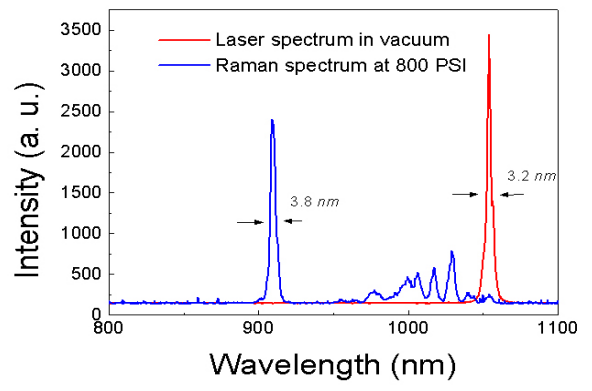

FIGURE 2. Comparison of the laser spectrum and the Raman spectrum at a helium gas pressure of 800 PSI. The Raman peak wavelength is $905 \mathrm{~nm}$. The intensity of Raman signal and laser are not in the same scale.

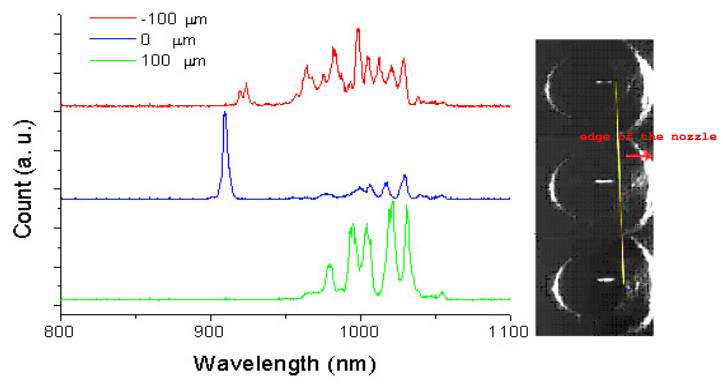

FIGURE 3. The Raman satellite signals at different focus position relative to the gas jet edge. $-100 \mu \mathrm{m}$ means the focus position is $100 \mu \mathrm{m}$ away from the edge of the gas jet. The Raman signal merges when focus position is at $100 \mathrm{um}$ away from the gas jet edge, is strongest at gas jet edge and disappears when laser focus position is $100 \mu \mathrm{m}$ inside the gas jet.

increased and the bandwidth of Raman spectrum got broadened. The modulated side bands can be observed on Figure 4 at a laser power of 2.0 TW. When the laser power was increased to $2.4 \mathrm{TW}$, the Raman spectrum showed the character of asymmetric broadening with higher intensity on the red-shifted side. 


\begin{abstract}
ANALYSIS
The sensitivity of the Raman generation relative to the laser focal position can be explained by the ionization blue-shifting seeding mechanism. When the laser is focused at the edge of the gas jet, the self-ionization front copropagated with laser pulse and laser frequency can be kept upshifted. While the laser is focused inside the gas jet, as the result of temporal and spatial distribution of the laser intensity, the ionization front propagates back and keeps encountering the original laser frequency[8], so the laser frequency can not be kept blue-shifted. The laser phase-front is also disturbed during the back-propagating ionization process. Even the laser still can copropagate with the ionization front after the focus postion, the Raman signal cannot grow efficiently due to the distortion of the phase-front. This phenomena has been observed experimentally[9] and explains the disappearance of the Raman signal when the laser focus is inside the gas jet. Though the ionization defocusing can prevent the laser pulse from reaching its full intensity (that which it would reach in vacuum after being focused), it doesn't affect our particular experiment since helium is a low $\mathrm{Z}$ gas and more than $65 \%$ of the laser energy is inside the ionization contour at the laser power of $0.45 \mathrm{TW}$. This means that only the front of laser is defoused by the ionization effect, and the main pulse will still propagate through a uniform density plasma without defocusing since helium has been fully ionized.
\end{abstract}

The blue shift frequency can be calculated from the blue shift formula for laser selfionized front[10],

$$
\omega(\tau)=\omega_{0}\left(1+2 c \tau \omega_{p 0}^{2} / L_{0} \omega_{0}^{2}\right)^{\frac{1}{2}}
$$

The laser propagation distance $c \tau$ is the rayleigh range, which is approximately $200 \mu \mathrm{m}$ with our experimental parameters. $L_{0}$ is the ionization front length which can calculate from the time for fully ionizing the helium. From Ammosov, Delone, and Krainov (ADK) model[11] for tunnelling ionization, or from the ionization rate in Ref [12], the fully ionization time of helium is about $40 \mathrm{fs}$ for $400 \mathrm{fs}$ Gaussian laser profile with peak density of $2.4 \times 10^{17} \mathrm{Wcm}^{-2}$. From Equation 1, the final blue-shift frequency is $1.35 \omega_{0}$, which is much higher than the Raman frequency $1.16 \omega_{0}$. So the ionization blue-shifted spectrum of laser has enough bandwidth to seed the Raman signal. The sensitivity of FRS with the relative position of the laser to the gas jet also can rule out the Raman backscattering seeding mechanism since the Raman backscattering grows from thermal noise and should not be affected by the focus position. The spectrum resolved top image in the Figure 5 also confirmed the analysis. Figure 5 shows that the spectrum was kept blue shifted along laser propagation direction at laser power of $0.45 \mathrm{TW}$. When the laser peak intensity is increased to $2.4 \mathrm{TW}$, the Thompson scattering of anti-stoke Raman satellite can be observed on the top view spectrum. Because of the ionization induce scattering instability[13], the scattering light observed on the top view are mainly from the ionization front. The observation indicates that the intensity of Raman signal grow much stronger than that of other wavelength. Due to its faster group velocity, Raman signal exceeds the laser pulse and ionizes the helium gas .

Though the broadening of the Raman spectrum shown in Figure 4 was previously attributed to plasma-wave wavebreaking [14], we found it is actually due to the relativistic cross-phase modulation (XPM) for our particular experimental parameters. The XPM 


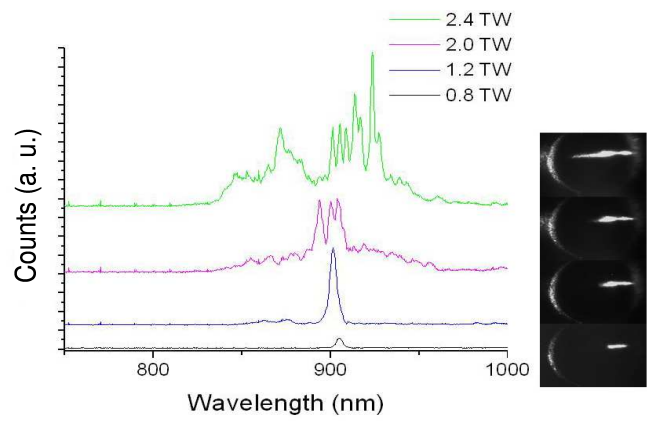

FIGURE 4. Raman spectrum at different laser energies. The inset picture shows the channel length of the laser plasma interaction. The self-focusing begin to happen at a laser intensity of $1.2 \mathrm{TW}$.

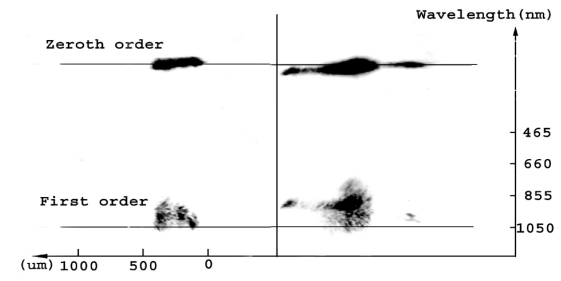

FIGURE 5. The spectrum resolved topview image. The picture on the left side is the top view image at laser power $0.45 \mathrm{TW}$. It shows that the laser spectrum kept blue shifted as laser propagates. picture at right side at the laser power of $2.4 \mathrm{TW}$ shows the Raman grows from the blue shifted light and the intensity is strong enough to ionize the helium gas.

refers to the phase modulation of one optical field by another copropogating optical field of a different wavelength. It can be described by the coupled nonlinear Schrödinger equation[15]:

$$
\begin{gathered}
\frac{\partial A_{p}}{\partial z}+\frac{i}{2} \beta_{2 p} \frac{\partial^{2} A_{p}}{\partial T^{2}}=i \delta_{p}\left(\left|A_{p}\right|^{2}\right) A_{p} \\
\frac{\partial A_{s}}{\partial z}+d \frac{\partial A_{s}}{\partial z}+\frac{i}{2} \beta_{2 s} \frac{\partial^{2} A_{s}}{\partial T^{2}}=i \delta_{s}\left(2\left|A_{p}\right|^{2}\right) A_{p}+\frac{g_{s}}{2}\left|A_{p}\right|^{2} A_{s}
\end{gathered}
$$



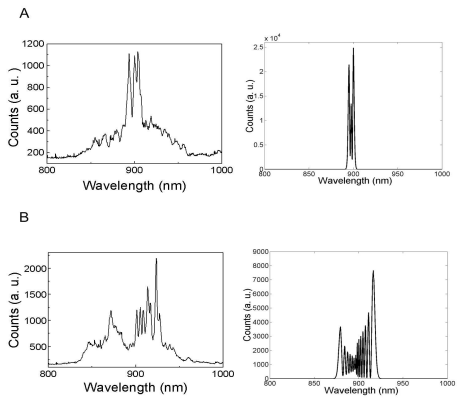

FIGURE 6. The comparison of experimental data and simulation results, showing good agreement between the observation and theoretical predictions. (a) The XPM of Raman at 2.0 TW. The laser power used in the simulation is $2 \mathrm{TW}$, and propagation distance is $200 \mu \mathrm{m}$. (b) The XPM of Raman at 2.4 TW. The laser power used in simulation is $2.4 \mathrm{TW}$ and propagation distance is $800 \mu \mathrm{m}$.

where

$$
T=t-z / v_{g p}, d=\left(v_{g p}-v_{g s}\right) /\left(v_{g p} v_{g s}\right), \delta=n_{2} \omega_{0} /\left(c A_{e f f}\right), \beta_{2}=\frac{\omega}{c} \frac{d^{2} n}{d \omega^{2}} .
$$

In the equations, $A$ is the electric field envelope amplitude of the optical pulse. $T$ is the time measured in the moving frame of pump pulse. $g_{s}$ is the Raman gain coefficient. $\delta$ is the nonlinear coefficient and $\delta_{p}=1.32 \times 10^{-11} \mathrm{~m}^{-1} \mathrm{~W}^{-1}, \delta_{s}=1.53 \times 10^{-11} \mathrm{~m}^{-1} \mathrm{~W}^{-1}$ in our experiment. $d$ is the walk-off parameter by which we can define the walk-off distance $L_{W}=T_{0} /|d|$, which determines the importance the first derivative in the equation (3). With the experimental parameters $d=2.0 \times 10^{-11} \mathrm{~s} / \mathrm{m}$ and $T_{0}=400 / 1.665=240$ fs, the walk off distance $L_{W}$ is equal to $1.2 \mathrm{~cm}$. $\beta_{2}$ is the dispersion parameter, and $\beta_{2 p}=2.2 \times 10^{25} \mathrm{~s}^{-2}, \beta_{2 s}=3.1 \times 10^{25} \mathrm{~s}^{-2}$. The dispersion distance, $L_{D}=T_{0} /\left|\beta_{2}\right|$, is defined to determine the importance of the second derivative in the Equations (2) and (3). The second derivative in the equation can be neglected if the dispersion distance is much larger than the interaction distance. From the dispersion parameter calculated above, the dispersion distance for the laser and Raman are about $78 \mathrm{~cm}$ and $108 \mathrm{~cm}$. Since the interaction distance $L$ is about $1 \mathrm{~mm}$, we have $L<L_{W} \ll L_{D}$ and the second derivative term in the coupled equation can be neglected. With simplification, the solution of the coupled equations can be written as:

$$
\begin{array}{r}
A_{p}(L, T)=A_{p}(0, T) \exp \left(i \delta_{p} \phi_{p}\right), \\
A_{s}(L, T)=A_{s}(0, T-L d) \exp \left(\left(g_{s}+i \delta_{s}\right) \phi_{s}\right), \\
\phi_{p}=P_{0} \exp \left(-\tau^{2}\right) L \\
\phi_{s}=P_{0} \frac{\sqrt{\pi}}{\tau}[\operatorname{erf}(\tau+\delta)-\operatorname{erf}(\tau)] L
\end{array}
$$

The theoretical spectrum were plotted using the experimental parameters. To obtain good agreement for the cross-modulated peak's bandwidth, the input laser pulse duration 
